\title{
A Compact Polarization-Independent Wavelength Duplexer Using a Polarization-Diversity SOI Photonic Wire Circuit \\ Wim Bogaerts ${ }^{1}$, Dirk Taillaert ${ }^{1}$, Pieter Dumon ${ }^{1}$, Elroy Pluk ${ }^{2}$, Dries Van Thourhout ${ }^{1}$, Roel Baets \\ ${ }^{1}$ Ghent University - Interuniversity Microelectronics Center (IMEC), Department of Information Technology \\ Sint-Pietersniewstraat 41, 9000 Gent, Belgium Wim.bogaerts@intec.ugent.be \\ ${ }^{2}$ Genexis B.V., Lodewijkstraat 1a, 5652 AC Eindhoven, The Netherlands
}

\begin{abstract}
We present a wavelength duplexer in silicon-on-insulator photonic wires. We made a polarization-diversity circuit using fiber grating couplers with polarization splitter and a bidirectional AWG, resulting in a polarization dependent loss of only $0.66 \mathrm{~dB}$. (C)2006 Optical Society of America

OCIS codes: (130.0130) Integrated Optics; (130.1750) Components; (230.3120) Integrated Optics Devices
\end{abstract}

\section{Introduction}

Silicon-on-insulator nanophotonic waveguides promise a dramatic reduction in size for photonic integrated circuits [1]. This could lead to both larger-scale integration and inexpensive components. These are a requirement for the wide deployment of optical access networks. While a number of photonic building blocks, based on low-loss nanophotonic waveguides, have already been demonstrated using commercial fabrication tools [1], there are still a number of obstacles for the widespread adoption of this technology. Because of their high index contrast and wavelength-scale dimensions, photonic wires are inherently polarization dependent, so circuits can only be designed for a single polarization. On the other hand, in most optical fiber links the polarization is an unknown and changing quantity. A solution is using a polarization-diversity scheme, where the polarization in the fiber is split into two orthogonal polarizations which are processed independently in separate circuits [2]. This way, polarizationindependent operation can be achieved.

We demonstrate a polarization-diversity approach which is based on a compact fiber coupler grating with integrated polarization splitter [3]. As an application, we implemented a wavelength duplexer for a low-cost fiber access network using a very compact arrayed waveguide grating (AWG).

\section{Wavelength duplexer}

Many of today's fiber-to-the-home (FTTH) systems employ 2 different wavelengths for upstream and downstream traffic. Typically, these wavelengths are in widely separated around $1310 \mathrm{~nm}$ and $1550 \mathrm{~nm}$. However, there is an emerging trend towards a wavelength division multiplexer (WDM) approach over a passive optical network (PON), where multiple wavelengths for multiple subscribers can be used over the same fiber. An important aspect of this approach is that while the subscribers use different wavelengths, they all use the same transceiver module, which therefore should be wavelength-independent for all upstream and all downstream wavelengths. To realize this, often reflective modulation is used (e.g. by a reflective SOA like in Fig.1) on a downstream-travelling CW carrier. In this case, all wavelength allocation can be done in the central office [4][5]. One possible choice for upstream and downstream wavelengths is to group them in two wider wavelength bands. In this work, we used two $350 \mathrm{GHz}$ bands, each containing 8 x 50GHz channels, with a band separation of $150 \mathrm{GHz}$.

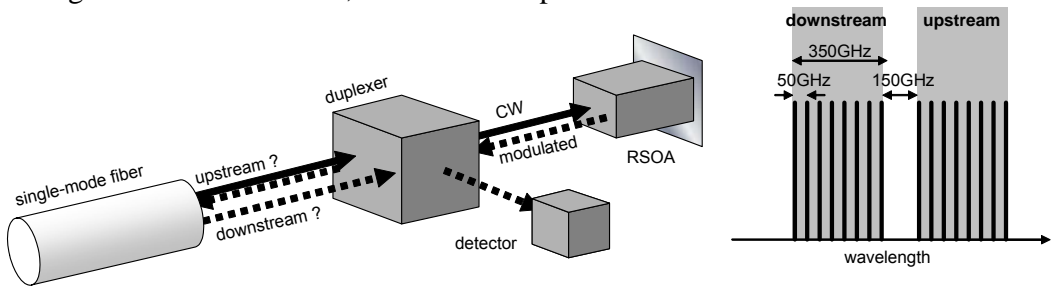

Fig. 1. Principle of duplexer-based fiber access end-point. The downstream and upstream operate each on one 50GHz-spaced ITU channel within a $350 \mathrm{GHz}$ band. By using a reflective approach, the channel can be selected at the central office.

For this purpose, we designed a wavelength duplexer using an arrayed waveguide grating. Photonic wires in silicon-on-insulator make it possible to implement very compact wavelength-selective functions. The main challenge in this design is the definition of two broad wavelength bands with a fairly small channel spacing. We solve this by using two AWG channels for each wavelength band. The resulting AWG contains 48 arrayed waveguides and requires a footprint of $600 \mu \mathrm{m} \times 350 \mu \mathrm{m}$ (Fig 3). The AWG was designed with a nominal channel 


\section{OTuM2.pdf}

spacing of $275 \mathrm{GHz}$, and a free spectral range of 3.85THz. 48 arrayed waveguides were used. However, the input and 4 output ports were customized to meet the design requirements of $2 \times 350 \mathrm{GHz}$ bands.

To increase coupling efficiency in the star couplers of the AWG, a two-step etch process was used, where the interface between access waveguides and slab region has a lower index contrast to reduce reflections.

\section{Polarization-Diversity with Grating Fiber Couplers}

The main challenge of using photonic wires is to maintain polarization independence when interfacing to the fiber. Fiber couplers gratings, as now often used for SOI nanophotonic circuits, typically work for a single fiber polarization. However, by using a 2-D periodic grating, both fiber polarizations can be coupled to the TE-mode of their own waveguides [3]. This way, the grating coupler also functions as a polarization splitter, making a polarization diversity approach possible by processing both fiber polarizations in their own circuit. And as both fiber polarizations are coupled to the TE-mode of the waveguide, these circuits can be designed identical, obviating the need for polarization conversion.

To ensure polarization independence when using this approach, both circuits should function identical. For wavelength-selective functions this requires very accurate fabrication technology. Typically, to assure a $50 \mathrm{GHz}$ accuracy $(0.4 \mathrm{~nm})$ in channel positioning, the dimensions of the waveguide should be controlled to within $1 \mathrm{~nm}$ or better. While we used advanced CMOS processing for the fabrication, the reproducibility and uniformity is insufficient to guarantee this accuracy. This could be solved with active tuning mechanisms, but this would dramatically increase the complexity of the device.

An elegant solution to this problem is by propagating both fiber polarizations through the same circuit. In an AWG, the critical element that controls the channel spacing is the array of delay lines. We designed our AWG such that both polarizations could use the same set of delay lines by propagating in opposite directions. This principle is shown in Fig. 2. The two orthogonal fiber polarizations are indicated with $P 1$ and $P 2$. For the first demonstration of the duplexer, we have not added the detector and RSOA, but directed the output to similar 2-D fiber couplers, where the outputs are combined into orthogonal fiber polarizations.

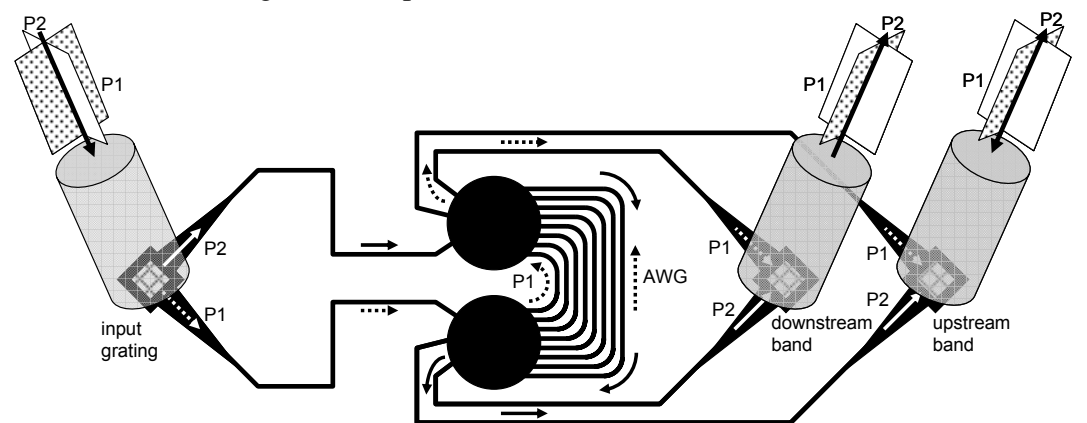

Fig. 2. Principle of a polarization independent duplexer using a polarization splitting grating coupler and bidirectional propagation through an AWG. Both fiber polarization travel through the same set of grating arms in opposite direction. At the output the polarizations can be recombines using the same type of 2-D grating coupler.

\section{Fabrication and Results}

The duplexer was fabricated with CMOS-compatible processes on commercial 200mm silicon-on-insulator wafers with a $220 \mathrm{~nm}$ silicon top layer and $2 \mu \mathrm{m}$ oxide. Patterning was done with 248nm Deep UV lithography [1]. The etching was done in two steps. First, a shallow etch of $70 \mathrm{~nm}$ is used for the AWG star couplers and the 2-D fiber couplers. Next, the waveguides are defined using a deep etch through the SOI core. The device is shown in Fig. 3.

We characterized the device using a broadband LED and an optical spectrum analyser. Both are connected to standard single-mode fiber which is compatible with the 2-D grating couplers. Fig. 4a shows the transmission of a reference waveguide connecting two 2-D fiber coupler gratings. The loss for a single fiber-to-chip coupling has a Gaussian wavelength dependence, with an optimal efficiency of $21 \%$ (-6.7dB), and a $3 \mathrm{~dB}$ bandwidth of $60 \mathrm{~nm}$.

Fig. 4b shows the output of the four AWG channels normalized to the reference waveguide. In the final device, the four AWG output channels will be combined in pairs into the two $350 \mathrm{GHz}$ wavelength bands indicated in Fig. 4b. Nonuniformity within these combined wavelength bands is $3.4 \mathrm{~dB}$, with an insertion loss between $-2.2 \mathrm{~dB}$ and $5.6 \mathrm{~dB}$. Crosstalk between the bands is as low as $-15 \mathrm{~dB}$. While the shape and size of the transmission bands matches the design values, their absolute positioning is not well controlled. This would require tuning of the circuit. 


\section{OTuM2.pdf}

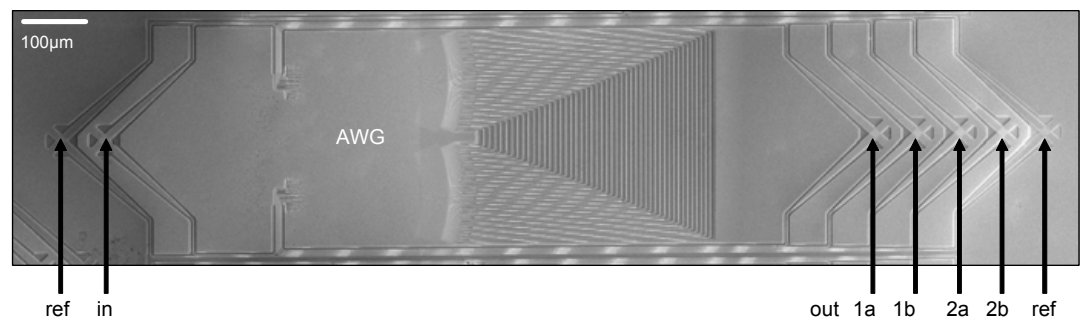

Fig. 3. SEM of fabricated duplexer. The outermost fiber couplers are directly connected and are used for as alignment and reference. The AWG has a $600 \times 350 \mu \mathrm{m}^{2}$ footprint. The two outputs for each wavelength band are not grouped but characterised individually.

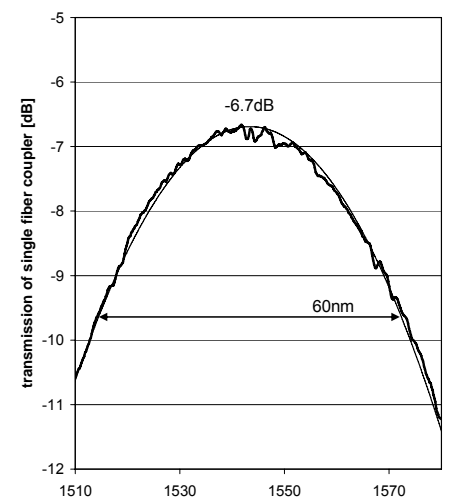

(a)

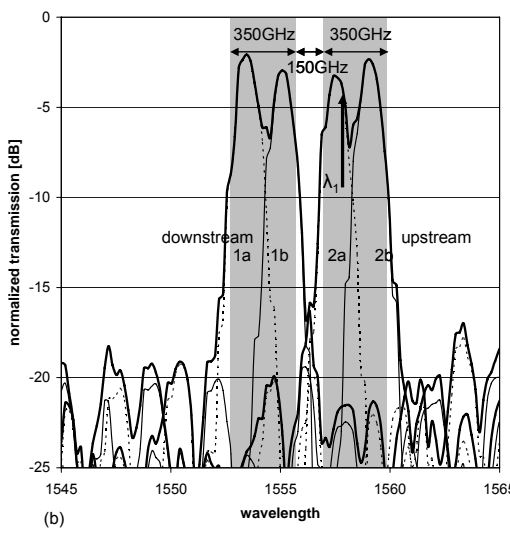

(b)

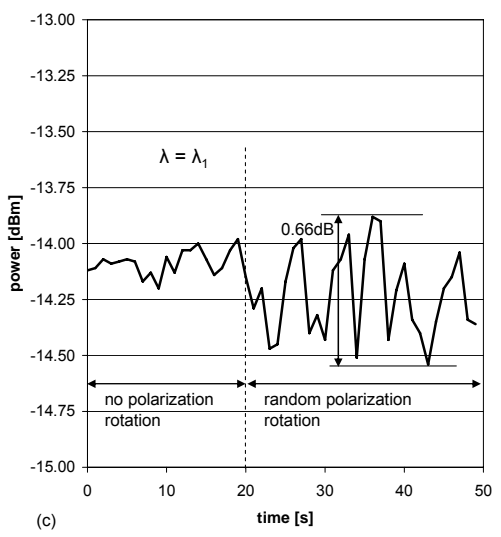

Fig. 4. Measurements. (a) transmission spectrum of a single 2-D grating coupler. (b) Transmission of the 2 duplexer output pairs indicated in Fig. 3 normalized to the transmission of the grating couplers.

To measure polarization dependence, we observed the output power while randomly varying the input polarization. Fig. 3c shows the power fluctuation over time with and without polarization rotation. The wavelength was kept constant near the peak of port 2a. For stabilization of input and output fiber, index-matching fluid was applied, which also enhanced the transmission. We measured a polarization dependent loss (PDL) of only $0.66 \mathrm{~dB}$.

\section{Conclusion}

We have demonstrated a compact, polarization-independent wavelength duplexer in silicon-on-insulator photonic wire waveguides. Using a compact 2-D diffraction grating, we can couple to standard single-mode fiber while at the same time split the unknown fiber polarization into two separate circuits. To ensure polarization independent behaviour, we propagate both polarizations through an arrayed waveguide grating in opposite direction. Measurements show well-defined bands for upstream and downstream wavelength, with crosstalk of $-15 \mathrm{~dB}$, and a polarization dependent loss of $0.66 \mathrm{~dB}$.

\section{Acknowledgements}

This work was partly supported by the European Union through the IST-PICMOS project, the IST-ePIXnet Network of Excellence and the Silicon Photonics Platform, and partly supported by the Belgian IAP-PHOTON project.

\section{References}

[1] W. Bogaerts, R. Baets, P. Dumon, V. Wiaux, S. Beckx, D. Taillaert, B. Luyssaert, J. Van Campenhout, P. Bienstman, and D. Van Thourhout, “Nanophotonic waveguides in Silicon-on-insulator fabricated with CMOS technology,” J. Lightw. Technol. 23 (1), p. 401 , 2005.

[2] C.D. Doerr, M. Zirngibl, C.H. Joyner, L.W. Stultz, H.M. Presby, "Polarization diversity Waveguide Grating Receiver with Integrated Optical Amplifiers”, IEEE Photon. Technol. Lett. 9, pp. 85, 1997.

[3] D. Taillaert, H. Chong, P. Borel, L. Frandsen, R.M. De La Rue, R. Baets, “A compact two-dimensional grating coupler used as a polarization splitter”,IEEE Photon. Technol. Lett. 15(9), p.1249, 2003

[4] P. J. Urban, E. G. C. Pluk, E. J. Klein, A. M. J. Koonen, G. D. Khoe, H.de Waardt, "Simulation Results of Dynamically Reconfigurable Broadband Photonic Access Networks (BB Photonics)", 2nd IET International Conference on Access Technologies ICAT, p. 93, Cambridge, UK, Jul. 2006

[5] R. Roy, G. Manhoudt, C. Roeloffzen, W. van Etten, "Control and Management Scheme in a DWDM EPON", Proceedings of the 8th International Conference on Transparent Optical Networks ICTON 2006, p. Tu. D1.6, Nottingham, UK, June 2006 